\title{
The Reorientation Temperature in an Antiferromagnetic Monolayer
}

\author{
V. ILKOVICั $\check{C}^{a}$ AND J. KECER ${ }^{b}$ \\ ${ }^{a}$ Institute of Physics, Faculty of Science, P.J. Šafarik University, Jesenná 5, 04001 Košice, Slovak Republic \\ ${ }^{b}$ Department of Physics, Technical University, Park Komenského 2, 04001 Košice, Slovak Republic \\ The reorientation temperature in an antiferromagnetic square lattice is studied within the framework of \\ many-body Green function theory. The model Hamiltonian includes a Heisenberg term, second-order single-ion \\ anisotropy, and a transverse external magnetic field in the $x$-direction. We investigate the orientation temperature \\ as a function of the single-ion anisotropy parameter and for different transverse magnetic fields.
}

PACS numbers: 75.10.Jm, 75.30.Ds, 75.30.Kz, 75.70.Ak

\section{Introduction and fundamental equations}

Recently, the Green function theories (GFT) [1-4] were proposed to treat the problem of magnetic reorientation phase transition (MRPT) in ultrathin magnetic films. In our consideration of MRPT in an antiferromagnetic monolayer (ML) we use GFT developed by Schwieger et al. [2] for ferromagnetic thin films. Our aim in this paper is to study the reorientation temperature $T_{\mathrm{R}}$ as a function of the surface single-ion anisotropy parameter $K_{2}(T \rightarrow 0)$ and of the transverse magnetic field $h_{0}^{x}$. In order to study the field-induced magnetic reorientation, the Hamiltonian can be written as

$$
\begin{aligned}
\mathcal{H} & =\frac{1}{2} J \sum_{\langle i j\rangle}\left(S_{i}^{-} S_{j}^{+}+S_{i}^{z} S_{j}^{z}\right)+\frac{1}{2} D \sum_{\langle i j\rangle} S_{i}^{z} S_{j}^{z} \\
& -\sum_{i} K_{2}(0)\left(S_{i}^{z}\right)^{2}-h_{0}^{x} \sum_{i} S_{i}^{x} .
\end{aligned}
$$

The first term is the Heisenberg operator for exchange energy with $J>0$ and $D$ denotes an anisotropy constant. The third term represents the single-ion anisotropy with the parameter $K_{2}>0$. The transverse magnetic field $h_{0}^{x}$ is shorthand for $g \mu_{\mathrm{B}} H^{x}$.

The theory in [2] is based on a transformation of the fixed coordinate system $(x, y, z)$ into a local coordinate system $(X, Y, Z)$. The magnetization in the fixed system $(x, y, z)$ can be read as

$$
\left\langle S^{x}\right\rangle=\left\langle S^{Z}\right\rangle \sin \theta, \quad\left\langle S^{z}\right\rangle=\left\langle S^{Z}\right\rangle \cos \theta,
$$

where $\theta$ is the angle between the $z$ axis and the magnetization.

A remarkable result of this theory is that the effective field aligned parallel to the magnetization can be written as a sum of the external magnetic field and an anisotropy field

$$
h=h_{0}^{x} \sin \theta+2 K_{2}(0)\left[\cos ^{2} \theta-\left(\sin ^{2} \theta\right) / 2\right]\left\langle S^{Z}\right\rangle Q^{(S)}
$$

with $Q^{(S)}=1-\left[S(S+1)-\left\langle\left(S^{Z}\right)^{2}\right\rangle\right] / 2 S^{2}$.

The orientation angle of the magnetization in $\mathrm{ML}$ is determined by the components of the effective field

$$
\tan \theta=\frac{h^{x}}{h^{z}}=\frac{h_{0}^{x}-K_{2}(0)\left\langle S^{Z}\right\rangle \sin ^{3} \theta Q^{(S)}}{2 K_{2}(0)\left\langle S^{Z}\right\rangle \cos \theta\left[1-\left(\sin ^{2} \theta\right) / 2\right] Q^{(S)}} .
$$

In the antiferromagnetic case, the square lattice is assumed to be divided into two interpenetrating sublattices. We now introduce sublattice indices $(n, m)$ for the up $(A)$ and down $(B)$ spins. Four equations of motion for the Green functions (GF) in the local reference frame: $G_{i_{n} j_{m}}(\omega)=\left\langle S_{i_{n}}^{+} ;\left(S_{j_{m}}^{Z}\right)^{l} S_{j_{m}}^{-}\right\rangle_{\omega}$ corresponding to the pairs $\left(i_{n}, j_{m}\right)=(A, A),(B, A),(A, B)$ and $(B, B)$ result. The exponent $l$ is a positive integer number $(0 \leq l \leq 2 S)$ defined. The subscripts of the GFs in momentum space $G_{m n}(\omega \boldsymbol{q})$ denote sublattice indices. As $\left\langle S^{Z}\right\rangle_{B}=-\left\langle S^{Z}\right\rangle_{A}$ for an antiferromagnet, the four equations of motion decouple to two identical pairs of equations which determine $\left\langle S^{Z}\right\rangle_{A}$ or $\left\langle S^{Z}\right\rangle_{B}$, respectively.

We use the technique of the equation of motion for the Green functions within the usual random phase approximation (RPA) for the Green function appearing in the nonlocal exchange term and a generalized Anderson-Callen approximation developed by Schwieger et al. [2] and applied to investigate the reorientation temperature in ferromagnetic films [5], in the local anisotropy term. After decoupling procedures for the Fourier components of the Green function $G_{A A}(\omega, \boldsymbol{q})$ we have the following equation:

$$
\begin{aligned}
& G_{A A}(\omega, \boldsymbol{q})=\frac{Z_{A}^{(l)}}{\omega^{+}-\omega^{-}}\left(\frac{\omega^{+}-J_{0}\left\langle S^{Z}\right\rangle_{A}+h_{0}}{\omega-\omega^{+}}\right. \\
& \left.+\frac{\omega^{-}-J_{0}\left\langle S^{Z}\right\rangle_{A}+h_{0}}{\omega-\omega^{-}}\right)
\end{aligned}
$$

where

$$
\begin{aligned}
& Z_{A}^{(l)}=2\left\langle\left[S^{+},\left(S^{Z}\right)^{l} S^{-}\right]\right\rangle_{A}, \\
& \omega^{ \pm}=-h_{0} \pm\left\langle S^{Z}\right\rangle_{A} \sqrt{J_{0}^{2}-J_{\boldsymbol{q}}^{2}},
\end{aligned}
$$




$$
\begin{aligned}
J_{0} & =-J\left[4(1+D / J)-2\left(K_{2} / J\right)\right. \\
& \left.\times\left\langle\left[\cos ^{2} \theta-\left(\sin ^{2} \theta\right) / 2\right]\right\rangle Q^{(S)}\right], \\
J_{\boldsymbol{q}} & =-4 J \cos \left(q^{x} / 2\right) \cos \left(q^{y} / 2\right), \quad h_{0}=h_{0}^{x} \sin \theta .
\end{aligned}
$$

After using the spectral theorem we finally obtain the correlation function $C_{A}^{(l)}=\left\langle\left(S^{Z}\right)^{l} S^{-} S^{+}\right\rangle_{A}$ for the sublattice $A$ as

$$
\left\langle\left(S^{Z}\right)^{l} S^{-} S^{+}\right\rangle_{A}=\left\langle\left[S^{+},\left(S^{Z}\right)^{l} S^{-}\right]\right\rangle_{A} \Phi,
$$

where

$$
\begin{aligned}
\Phi & =\frac{1}{\pi^{2}} \int_{0}^{\pi} \int_{0}^{\pi} \frac{1}{\omega^{+}-\omega^{-}}\left[\frac{\omega^{+}-J_{0}\left\langle S^{Z}\right\rangle_{A}+h_{0}}{\exp \left(\beta \omega^{+}\right)-1}\right. \\
& \left.+\frac{\omega^{-}-J_{0}\left\langle S^{Z}\right\rangle_{A}+h_{0}}{\exp \left(\beta \omega^{-}\right)-1}\right] \mathrm{d}^{2} q^{x} q^{y} .
\end{aligned}
$$

From (6) we obtain for $S \geq 1$ the system of algebraic equations for calculation of $\left\langle S^{Z}\right\rangle_{A}$. The components of the magnetization per site in the sublattice $A$ in the fixed system $(x, y, z): m_{A}^{x} \equiv\left\langle S^{x}\right\rangle_{A}$ and $m_{A}^{z} \equiv\left\langle S^{z}\right\rangle_{A}$ can be then calculated from (2) and the orientation angle of the magnetization can be obtained from (4).

\section{Results}

The reorientation temperature $T_{\mathrm{R}}$ is defined as the temperature, at which in the sublattices $A(B)$ the longitudinal magnetization $m_{A(B)}^{z} \equiv\left\langle S^{z}\right\rangle_{A(B)}$ vanishes, the transverse magnetization $m_{A(B)}^{x} \equiv\left\langle S^{x}\right\rangle_{A(B)}$ is nonzero

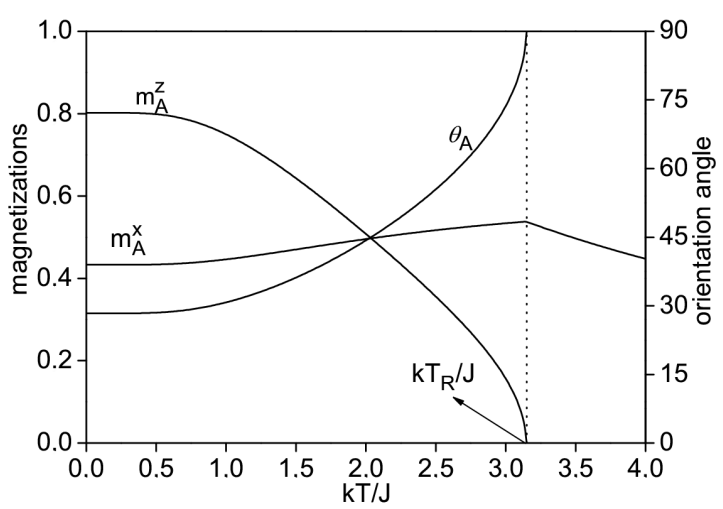

Fig. 1. The components of the sublattice magnetizations $m_{A}^{x}, m_{A}^{z}$ and the orientation angle $\theta_{A}$ as a function of the reduced temperature $k T / J$ are shown for an antiferromagnetic ML with $\operatorname{spin} S=1$.

and the equilibrium orientation angle $\left|\theta_{A(B)}\right|=90^{\circ}$. This can be seen in Fig. 1, where we plot the components of the magnetization per site $m_{A}^{x}$ and $m_{A}^{z}$ and the orientation angle $\theta_{A}$, as a function of the reduced temperature $k T / J$ for spin $S=1$ when the reduced single-ion anisotropy parameter at zero temperature $K_{2}(0) / J=0.01$. The external magnetic field is directed along the $x$-axis with

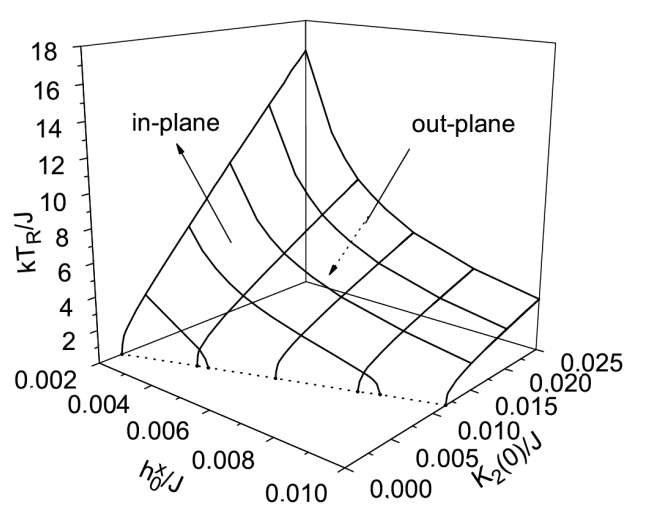

Fig. 2. The reduced reorientation temperature $k T_{\mathrm{R}} / J$ is shown as a function of the reduced transverse magnetic field in the $x$-direction $h_{0}^{x} / J$ and of the anisotropy parameter at zero temperature $K_{2}(0) / J$.

$h_{0}^{x} / J=0.004$. The exchange integral $J=1.0$ and the anisotropy constant $D / J=0.1$. The similar result was obtained in [6] within the many-body Green function theory. In Fig. 2, the reduced reorientation temperature $k T_{\mathrm{R}} / J$ is shown as a function of the reduced anisotropy strength at zero temperature $K_{2}(0) / J$ and of the reduced external magnetic fields in the $x$-direction $h_{0}^{x} / J$. The dashed line in the plane $\left(h_{0}^{x} / J, K_{2}(0) / J\right)$, on which the pairs of points $\left[\left(h_{0}^{x} / J\right)_{\mathrm{MRPT}},\left(K_{2}(0) / J\right)_{\mathrm{MRPT}}\right]$ occur, marks the boundary between two regions. For $h_{0}^{x} / J<\left(h_{0}^{x} / J\right)_{\mathrm{MRPT}}$ and for $K_{2}(0) / J>\left(K_{2}(0) / J\right)_{\mathrm{MRPT}}$ the direction of the magnetization is out of the plane of $\mathrm{ML}$, while in the opposite case the magnetization is in the plane of ML. To our knowledge, there are no investigations of this phase diagram. Our method can be applied to the antiferromagnetic thin films and ferrimagnetic systems.

\section{Acknowledgments}

Financial support of this work was provided by grant 1/0138/08, Grant Agency for Science, Slovak Republic and part of this work (V.I.) was carried at the Bogoliubov Laboratory of Theoretical Physics, JINR, Dubna.

\section{References}

[1] P. Fröbrich, P.J. Jensen, P.J. Kuntz, A. Ecker, Eur. Phys. J. B 18, 579 (2000).

[2] S. Schwieger, J. Kienert, W. Nolting, Phys. Rev. B 71, 024428 (2005).

[3] M.G. Pini, P. Politi, R.L. Stamps, Phys. Rev. B 72, 014454 (2005).

[4] H.-Y. Wang, C. Huang, M. Qian, E.-G. Wang, J. Appl. Phys. 95, 7551 (2004).

[5] V. Ilkovič, Phys. Status Solidi B 246, 2366 (2009).

[6] C. Huang, H.-Y. Wang, E.-G. Wang, Chin. Phys. Lett. 20, 1593 (2003). 